\title{
Association of diameter and wall stresses of tricuspid aortic valve ascending thoracic aortic aneurysms
}

\author{
Axel Gomez, BS, ${ }^{a}$ Zhongjie Wang, PhD, ${ }^{a}$ Yue Xuan, PhD, MD, ${ }^{a}$ Michael D. Hope, MD, ${ }^{b}$ \\ David A. Saloner, $\mathrm{PhD},{ }^{\mathrm{b}}$ Julius M. Guccione, $\mathrm{PhD},{ }^{\mathrm{a}}$ Liang $\mathrm{Ge}, \mathrm{PhD},{ }^{\mathrm{a}}$ and Elaine E. Tseng, $\mathrm{MD}^{\mathrm{a}}$
}

\section{ABSTRACT}

Objective: Ascending thoracic aortic aneurysms carry a risk of acute type A dissection. Elective repair guidelines are designed around size thresholds, but the 1-dimensional parameter of maximum diameter cannot predict acute events in small aneurysms. Biomechanically, dissection can occur when wall stress exceeds strength. Patient-specific ascending thoracic aortic aneurysm wall stresses may be a better predictor of dissection. Our aim was to compare wall stresses in tricuspid aortic valve-associated ascending thoracic aortic aneurysms based on diameter.

Methods: Patients with tricuspid aortic valve-associated ascending thoracic aortic aneurysm and diameter $4.0 \mathrm{~cm}$ or greater $(n=221)$ were divided into groups by 0.5 $\mathrm{cm}$ diameter increments. Three-dimensional geometries were reconstructed from computed tomography images, and finite element models were developed taking into account prestress geometries. A fiber-embedded hyperelastic material model was applied to obtain longitudinal and circumferential wall stress distributions under systolic pressure. Median stresses with interquartile ranges were determined. The Kruskal-Wallis test was used for comparisons between size groups.

Results: Peak longitudinal wall stresses for tricuspid aortic valve-associated ascending thoracic aortic aneurysm were 290 (265-323) kPa for size 4.0 to $4.4 \mathrm{~cm}$ versus 330 (296-359) $\mathrm{kPa}$ for 4.5 to $4.9 \mathrm{~cm}$ versus 339 (320-373) $\mathrm{kPa}$ for 5.0 to $5.4 \mathrm{~cm}$ versus $318(293-351) \mathrm{kPa}$ for 5.5 to $5.9 \mathrm{~cm}$ versus $373(363-449) \mathrm{kPa}$ for $6.0 \mathrm{~cm}$ or greater $(P=8.7 \mathrm{e}-8)$. Peak circumferential wall stresses were 460 (421-543) $\mathrm{kPa}$ for size 4.0 to $4.4 \mathrm{~cm}$ versus 503 (453-569) $\mathrm{kPa}$ for 4.5 to $4.9 \mathrm{~cm}$ versus 549 (430-588) $\mathrm{kPa}$ for 5.0 to $5.4 \mathrm{~cm}$ versus 540 (471-608) $\mathrm{kPa}$ for 5.5 to $5.9 \mathrm{~cm}$ versus 596 (506-649) kPa for $6.0 \mathrm{~cm}$ or greater $(P=.0007)$.

Conclusions: Circumferential and longitudinal wall stresses are higher as diameter increases, but size groups had large overlap of stress ranges. Wall stress thresholds based on aneurysm wall strength may be a better predictor of patient-specific risk of dissection than diameter in small ascending thoracic aortic aneurysms. (J Thorac Cardiovasc Surg 2022;164:1365-75)

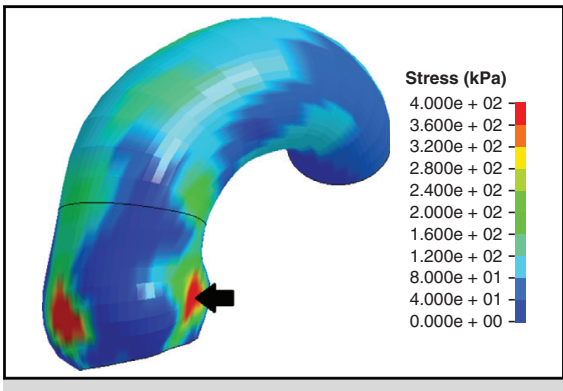

Longitudinal wall stress profile of small TAV-aTAA with high aortic root stress.

\section{CENTRAL MESSAGE}

Some small TAV-aTAAs have

peak wall stresses comparable to those of large aneurysms and are potentially at high risk of acute

\section{type A dissection.}

\section{PERSPECTIVE}

Type A dissection frequently occurs below aneurysm repair size thresholds. Diameter is a poor predictor of wall stress and thus dissection risk in aneurysms. Using patient-specific models, we found a subset of small aneurysms experiencing substantial wall stresses. FEA could be used to understand dissection risk and assist decisionmaking of elective surgical aTAA repair.

See Commentaries on pages 1376 and 1377 .

\footnotetext{
From the ${ }^{\mathrm{a}}$ Division of Adult Cardiothoracic Surgery, Department of Surgery, and ${ }^{\mathrm{b}}$ Department of Radiology \& Biomedical Imaging, University of California San Francisco and San Francisco VA Medical Center, San Francisco, Calif.

This study was funded by the National Institutes of Health (NIH) R01HL11985701A1 (L.G.), the American Heart Association Postdoctoral fellowship 20POST35211107 (Z.W.), and NIH K25HL150408 (Y.X.). This study was funded by NIH R01HL119857-01A1 (L.G., E.E.T.). CHR approval: 13-10932, 10-03594. Received for publication Dec 4, 2020; revisions received May 17, 2021; accepted for publication May 21, 2021; available ahead of print June 30, 2021.

Address for reprints: Elaine E. Tseng, MD, Division of Cardiothoracic Surgery, University of California San Francisco, San Francisco VA Medical Center, 500 Parnassus Ave, Suite 405W, San Francisco, CA 94143 (E-mail: Elaine.Tseng@ucsf.edu). 0022-5223

Published by Elsevier Inc. on behalf of The American Association for Thoracic Surgery. This is an open access article under the CC BY-NC-ND license (http:// creativecommons.org/licenses/by-nc-nd/4.0/).

https://doi.org/10.1016/j.jtcvs.2021.05.049
}

Ascending thoracic aortic aneurysm (aTAA) is often indolent and asymptomatic until dissection or rupture occurs. aTAA has a dissection or rupture rate of approximately $2 \%$ to $3 \%$ per year. $^{1}$ Aortic dissection and rupture are often fatal events in many patients before reaching the hospital, but still carry an approximately $25 \%$ operative mortality in patients undergoing emergency surgery. ${ }^{2}$ Annual incidence of dissection increases with aortic size and approaches $4 \%$ and $10 \%$ when greater than 5.5 and $6.0 \mathrm{~cm}$, respectively. $^{3}$ aTAA surgical repair is offered when maximum diameter reaches $5.5 \mathrm{~cm}$ or greater, with earlier intervention if rapid growth rate, family history of dissection, personal history of connective tissue disorder, or concurrent surgical aortic valve replacement is present. 


$$
\begin{aligned}
& \text { Abbreviations and Acronyms } \\
& \text { AscAo }=\text { ascending aorta } \\
& \text { aTAA }=\text { ascending thoracic aortic aneurysm } \\
& \text { BAV }=\text { bicuspid aortic valve } \\
& \text { CT }=\text { computed tomography } \\
& \text { FEA }=\text { finite element analysis } \\
& \text { LVOT }=\text { left ventricular outflow tract } \\
& \text { SOV }=\text { sinuses of Valsalva } \\
& \text { STJ }=\text { sinotubular junction } \\
& \text { TAV }=\text { tricuspid aortic valve } \\
& \text { 3D }=3 \text {-dimensional }
\end{aligned}
$$

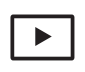

Scanning this QR code will take you to the table of contents to access supplementary information.

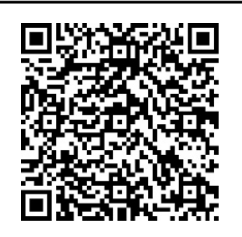

Current elective aTAA repair guidelines balance the risks of surgical intervention versus the risks of dissection. These diameter-based guidelines designed around numeric cutoffs use diameter as a surrogate for aneurysm wall stress based on the law of Laplace. However, diameter-based guidelines have failed to capture most small aTAAs at high risk of acute aortic events. Data from the International Registry of Acute Aortic Dissection as well as our data showed that approximately $60 \%$ of type A dissections had a diameter less than $5.5 \mathrm{~cm}$, and approximately $40 \%$ had a diameter less than $5 \mathrm{~cm}$ at the time of the event. ${ }^{5,6}$ Thus, acute aortic dissection is a complex hemodynamic event that is not well captured by diameter alone. There is unmet clinical need to develop an innovative approach to better predict acute aortic events and guide well-timed aTAA surgical repair.

From a biomechanical standpoint, dissection and rupture represent mechanical failure of the aortic wall. Mechanical failure can occur when wall stresses exceed wall strength. ${ }^{7}$ The law of Laplace linearly relates wall stress to both diameter and luminal pressure for spherical or cylindrical structures. However, aTAAs have complex geometries, and Laplace's law fails to accurately predict true aTAA wall stress. Fundamentally, this imperfect clinical correlation between aortic size and acute aortic events stems from using this simplified method to assess wall stress and thus patientspecific risk of dissection. Actual patient-specific aTAA wall stresses can be accurately calculated from 3dimensional (3D) computational models using finite element analysis (FEA), ${ }^{8,9}$ because wall stresses and failure strength are otherwise impossible to directly measure in vivo. FEA has successfully been applied to aTAAs and have shown that aortic regions with high wall stresses coincide with regions where initial intimal tears are observed. ${ }^{6,10,11}$ Studying aTAA wall stress profiles could improve detection of patients at high risk of acute aortic events and guide clinical decision-making. We have previously investigated wall stress versus diameter for a surgical threshold of less than $5 \mathrm{~cm}$ versus $5 \mathrm{~cm}$ or greater for both bicuspid aortic valve (BAV) and tricuspid aortic valve (TAV)-associated aTAAs because of our preference for operating on BAV at $5 \mathrm{~cm}$ or greater. ${ }^{12}$ That study had one of the largest sample sizes of aTAA FEA studies. In this study, we chose to focus only on TAV-aTAAs to determine wall stress magnitudes in relation to maximum aortic size and the impact of a $5.5-\mathrm{cm}$ surgical threshold.

\section{MATERIALS AND METHODS \\ Study Population}

Patients with TAV-aTAAs and measured diameter $4.0 \mathrm{~cm}$ or greater by computed tomography (CT) scan at San Francisco Veterans Affairs Medical Center between June 2007 and June 2020 were studied. We excluded patients with a BAV confirmed by echocardiography, CT, or surgery. This study was approved by the Committee on Human Research at University of California San Francisco Medical Center and Institutional Review Board at San Francisco Veterans Affairs Medical Center.

\section{Tricuspid Aortic Valve-Associated Ascending Thoracic Aortic Aneurysm Geometry Reconstruction}

De-identified CT scans were used to construct computational 3D models of TAV-aTAAs. Models included aortic regions from the left ventricular outflow tract (LVOT) to the mid-thoracic descending aorta. For each patient, CT images were imported into MeVisLab software (MeVis Medical Solutions AG, Bremen, Germany) for image segmentation to obtain a model of the aTAA surface. The surface data were imported into TrueGrid software (version 3.1.3; XYZ Scientific, Inc, Livermore, Calif) to create a geometric mesh model that was discretized into elements that resemble building blocks. Geometric meshes had 3 layers to reflect the intima, media, and adventitia layers with a total of 11,202 hexahedral elements. Meshes were imported into LS-DYNA R10 (LSTC Inc, Livermore, Calif) for finite element (FE) simulations and analysis.

\section{Finite Element Simulations}

When obtaining CT images, the aorta was under in vivo physiologic blood pressure conditions. Therefore, our initial models had a geometry that corresponded to systemic pressure and we corrected for prestresses to obtain a zero-pressure geometry suitable for FE simulations using a modified updateLagrangian method. ${ }^{13,14}$ FE simulations on TAV-aTAAs were done with a validated fiber-embedded hyperelastic material built and previously described by our group. ${ }^{14}$ Averaged TAV-aTAA material properties were applied based on previous biaxial stretch testing. ${ }^{15}$ The aortic wall was assumed to be incompressible, uniform, and hyperelastic. Aortic wall thickness was set at $1.8 \mathrm{~mm}$, derived from our prior aneurysm experimental studies. ${ }^{15}$ We fixed translational motion at the LVOT, $20 \mathrm{~mm}$ proximal to the aortic annulus, and at the mid-descending thoracic aorta, while rotational motion was unconstrained. These boundary conditions allowed for aortic motion with the cardiac cycle while accounting for restraints from the ligamentum arteriosum. The aortic root is a dynamic structure with the cardiac cycle causing displacement. Fixation at the LVOT did allow 3D displacement of the aortic root and aTAA as our particular regions of interest. TAV-aTAA models were subjected to physiologic luminal arterial pressures. The inner lumen was first pressurized to 
$80 \mathrm{~mm} \mathrm{Hg}$. The cardiac cycle was then simulated by gradually increasing luminal pressure from 80 to $120 \mathrm{~mm} \mathrm{Hg}$ over $300 \mathrm{~ms}$ followed by decreasing luminal pressure to $80 \mathrm{~mm} \mathrm{Hg}$ over $500 \mathrm{~ms}$. Video 1 shows the process of geometry reconstruction and FE simulations. Reproducibility was demonstrated with 2 investigators independently re-constructing TAV-aTAA models and performing FE simulations for a subset of 24 subjects.

\section{Statistical Methods and Data Analysis}

TAV-aTAA models were divided into 5 distinct groups based on the maximal diameter reported on their corresponding CTs. The groups were defined by aortic diameters of 4.0 to $4.4 \mathrm{~cm}, 4.5$ to $4.9 \mathrm{~cm}, 5.0$ to $5.4 \mathrm{~cm}, 5.5$ to $5.9 \mathrm{~cm}$, and $6.0 \mathrm{~cm}$ or greater. Statistical analysis was performed using 99th-percentile wall stresses, which have been shown to be more accurate by avoiding inhomogeneity in the mesh. ${ }^{16}$ Peak wall stresses will refer to 99th-percentile wall stresses. Peak wall stresses were calculated for both longitudinal and circumferential directions. Differences in peak stress between directions were studied with the paired samples $t$ test. The relationship of peak stress with aneurysm diameter was studied with the Kruskal-Wallis test to account for the non-normality of stresses in the different size groups. This analysis considered the whole aTAA model (ie, from the aortic annulus to the beginning of aortic arch). Secondary analyses on individual aortic regions were also performed, that is, sinuses of Valsalva (SOV), sinotubular junction (STJ), and ascending aorta (AscAo). Differences in peak stresses between blood pressures of 120 and $95 \mathrm{~mm} \mathrm{Hg}$ were studied with the Wilcoxon signed-rank test. Continuous variables are reported as median with interquartile range. Categorical variables are reported with count and percentages. Statistical analyses were performed using R (http://www.r-project.org). A flow diagram of the study methodology and primary outcomes is represented in Figure 1.

\section{RESULTS}

\section{Patient Demographics}

A total of 221 TAV-aTAA veterans were included in the current study with a median diameter of $4.5 \mathrm{~cm}$. The majority of patients were male $(97.3 \%)$ with a median age of 70 years. Hypertension and hyperlipidemia were the most common comorbidities, and veterans had a median 16 pack-year smoking history. Cardiovascular risk factors were distributed similarly among different aneurysm size groups with the exception of male sex $(P=.030)$. Table 1 presents demographic information.

\section{Circumferential Tricuspid Aortic Valve-Associated Ascending Thoracic Aortic Aneurysm Wall Stresses by Diameter}

Subjects were distributed into size groups based on maximal diameter. The number of aneurysms in groups 4.0 to $4.4 \mathrm{~cm}, 4.5$ to $4.9 \mathrm{~cm}, 5.0$ to $5.4 \mathrm{~cm}, 5.5$ to $6.0 \mathrm{~cm}$, and $6.0 \mathrm{~cm}$ or greater were $100,77,21,13$, and 10 , respectively. At systolic pressure, peak circumferential wall stresses were $460(421-543) \mathrm{kPa}$ for size 4.0 to $4.4 \mathrm{~cm}$, 503 (453-569) $\mathrm{kPa}$ for 4.5 to $4.9 \mathrm{~cm}, 549$ (430-569) $\mathrm{kPa}$ for 5.0 to $5.4 \mathrm{~cm}, 540(471-608) \mathrm{kPa}$ for 5.5 to $5.9 \mathrm{~cm}$, and 596 (506-649) $\mathrm{kPa}$ for $6.0 \mathrm{~cm}$ or greater (Table 2). Circumferential peak wall stresses increased with diameter $(P=.0008)$ (Figure 2, $A)$. The same trend was present for individual aTAA anatomic regions (Figure 2, $B-D$ ). Peak circumferential wall stresses for AscAo, STJ, and SOV are reported in Table 2. When diameter was evaluated as a continuous variable, the correlation of peak circumferential wall stresses to diameter was $\mathrm{r}=0.26(P=7.4 \mathrm{e}-5)$ for whole aTAA, $\mathrm{r}=0.61(P<2.2 \mathrm{e}-16)$ for AscAo, $\mathrm{r}=0.40$ $(P=3.7 \mathrm{e}-10)$ for STJ, and $\mathrm{r}=0.46(P=1.8 \mathrm{e}-13)$ for SOV, respectively.

\section{Longitudinal Tricuspid Aortic Valve-Associated Ascending Thoracic Aortic Aneurysm Wall Stress by Diameter}

At systolic pressure, peak longitudinal wall stresses were $290(265-323) \mathrm{kPa}$ for size 4.0 to $4.4 \mathrm{~cm}, 330(296-359) \mathrm{kPa}$ for 4.5 to $4.9 \mathrm{~cm}, 339(320-373) \mathrm{kPa}$ for 5.0 to $5.4 \mathrm{~cm}, 318$ (293-351) kPa for 5.5 to $5.9 \mathrm{~cm}$, and $373(363-449) \mathrm{kPa}$ for $6.0 \mathrm{~cm}$ or greater (Table 3$)$. Longitudinal peak wall stresses increased with diameter $(P=8.7 \mathrm{e}-8)$ (Figure $3, A)$. The same trend was present on individual aTAA anatomic regions (Figure 3, B-D). Peak longitudinal wall stresses for AscAo, STJ, and SOV are reported in Table 3. When diameter was evaluated as a continuous variable, the correlation of peak longitudinal wall stresses to diameter was $\mathrm{r}=0.38$ $(P=2.1 \mathrm{e}-9)$ for whole aTAA, $\mathrm{r}=0.51(P=3.4 \mathrm{e}-16)$ for AscAo, $\mathrm{r}=0.52(P<2.2 \mathrm{e}-16)$ for STJ, and $\mathrm{r}=0.52$ $(P<2.2 \mathrm{e}-16)$ for SOV, respectively. Representative wall stress profiles are shown in Figure 4.

\section{Circumferential Versus Longitudinal Tricuspid Aortic Valve-Associated Ascending Thoracic Aortic Aneurysm Wall Stress}

Peak wall stresses for TAV-aTAAs were greater in the circumferential than longitudinal direction (494 [432577] vs 313 [280-352] $\mathrm{kPa}, P<2.2 \mathrm{e}-16)$. The same relationship was true for TAV-aTAAs size 4.0 to $4.4 \mathrm{~cm}$ (460 [421-543] vs 290 [265-323] kPa, $P<2.2 \mathrm{e}-16)$, size 4.5

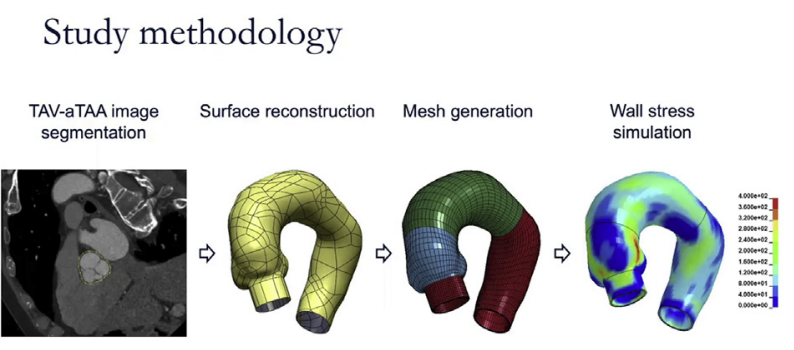

VIDEO 1. Schematic of study methodology displaying image segmentation of a representative TAV-aTAA, inner surface reconstruction (yellow), geometric mesh development with anatomic regions defined in color, and longitudinal wall stress profile with location of peak stress identified by filled arrow. Video also shows representative cardiac cycle simulation results for TAV-aTAA diameter groups. Color bar displays the relation of figure colors to wall stress magnitude in $\mathrm{kPa}$. Video available at: https:// www.jtcvs.org/article/S0022-5223(21)00909-0/fulltext. 
Relationship of Aneurysm Diameter and Wall Stress

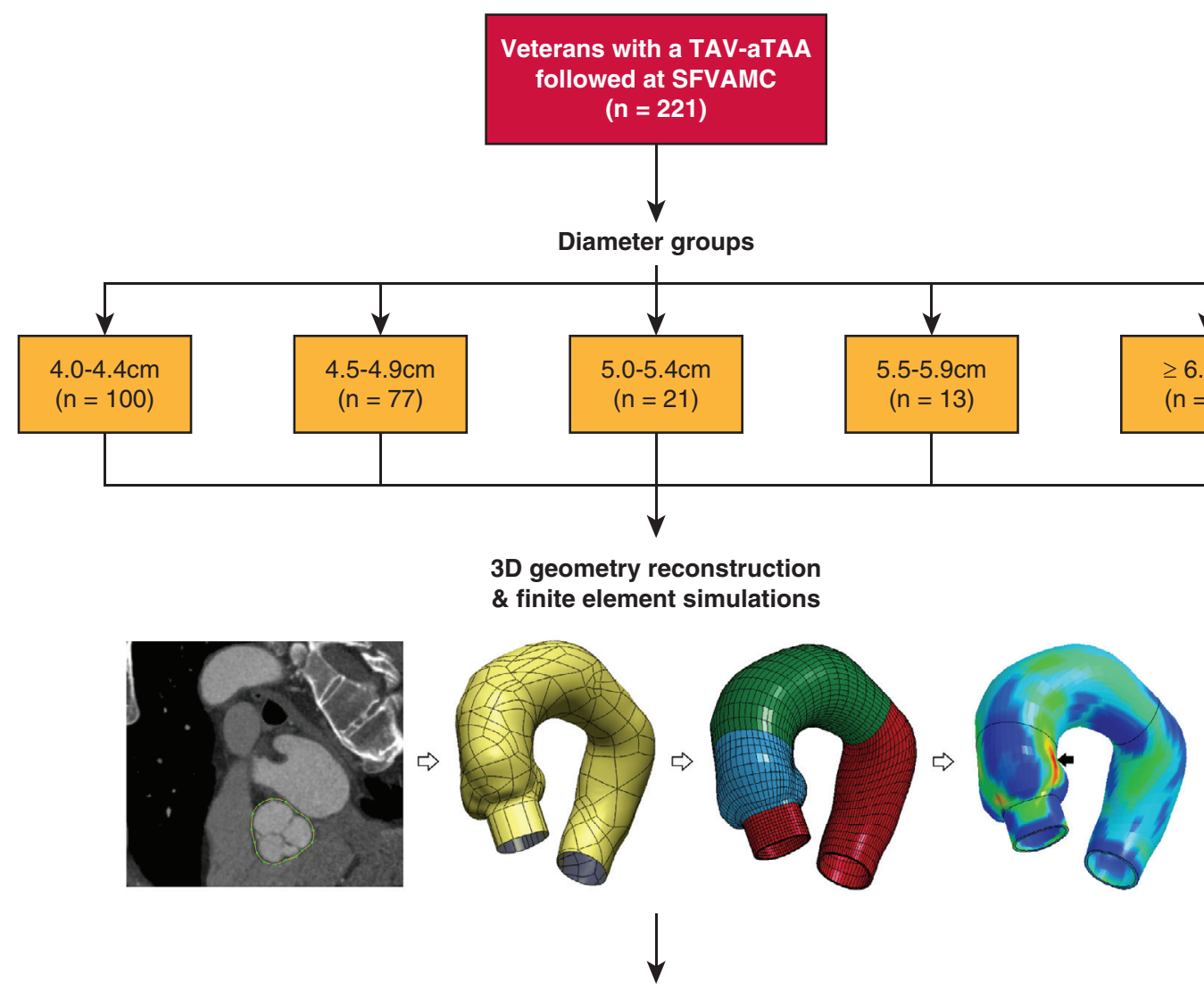

Peak circumferential wall stress

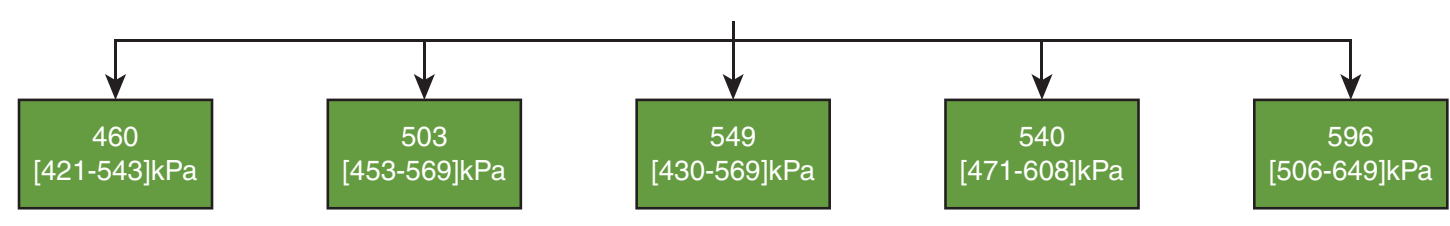

$+$

Peak longitudinal wall stress

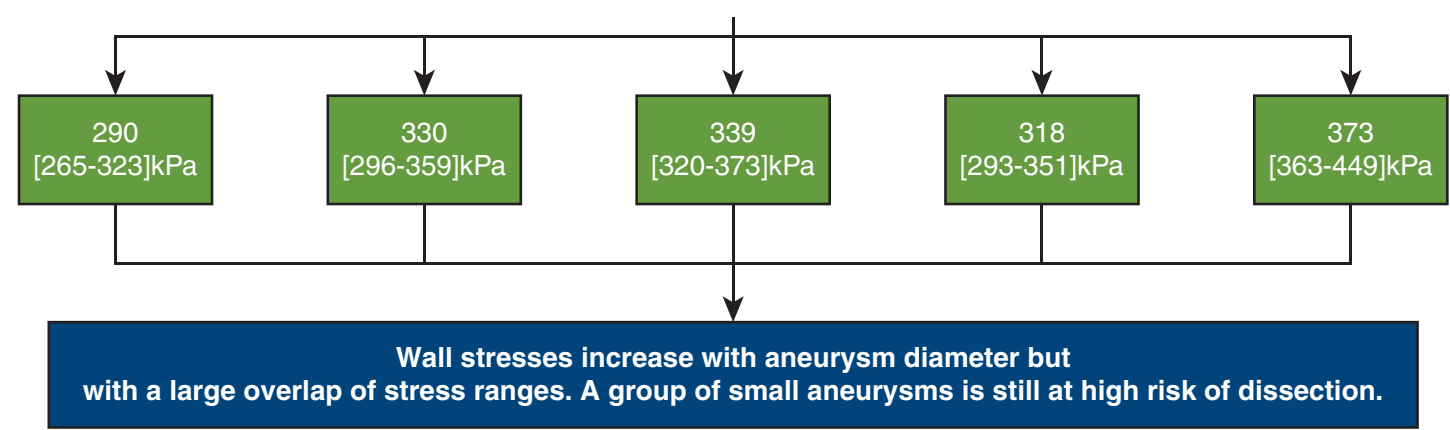

TAV-aTAA: Tricuspid aortic valve associated ascending thoracic aortic aneurysm SFVAMC: San Francisco Veterans Affairs Medical Center

FIGURE 1. Flow diagram representing TAV-aTAA sample population (first row), followed by stratification of patients into distinct groups by maximal aneurysm diameter (second row), schematic of aTAA model reconstruction and wall stress simulations (third row), measured peak circumferential (fourth row) and longitudinal (fifth row) wall stresses, and observed relationships between aneurysm peak wall stresses and diameter (sixth row). 
TABLE 1. Tricuspid aortic valve ascending thoracic aortic aneurysm population characteristics

\begin{tabular}{|c|c|c|c|c|c|c|c|}
\hline Characteristic & $4.0-4.4 \mathrm{~cm}(\mathrm{n}=100)$ & $4.5-4.9 \mathrm{~cm}(\mathrm{n}=77)$ & $5.0-5.4 \mathrm{~cm}(\mathrm{n}=21)$ & $5.5-5.9 \mathrm{~cm}(\mathrm{n}=13)$ & $\geq 6.0 \mathrm{~cm}(\mathrm{n}=10)$ & All $(n=221)$ & $P$ value \\
\hline Diameter $(\mathrm{cm})$ & $4.2(4.1-4.3)$ & $4.7(4.6-4.8)$ & $5.1(5.0-5.3)$ & $5.6(5.5-5.7)$ & $6.0(6.1-6.5)$ & $4.5(4.3-4.8)$ & $3.17 \mathrm{e}-40$ \\
\hline Age $(y)$ & $70(66-74)$ & $70(66-74)$ & $67(63-72)$ & $67(63-73)$ & $56(55-73)$ & $70(65-74)$ & .11 \\
\hline Male sex & $97(97.0 \%)$ & $77(100 \%)$ & $20(95 \%)$ & $11(85 \%)$ & $10(100 \%)$ & $215(97 \%)$ & .030 \\
\hline Smoking (pack-y) & $20(0-45)$ & $15(0.5-40)$ & $15(0-43)$ & $20(3-30)$ & $2(0-25)$ & $16(0-40)$ & .73 \\
\hline Hypertension & $78(78.0 \%)$ & $65(84.4 \%)$ & $18(85.7 \%)$ & $10(76.9 \%)$ & $7(70.0 \%)$ & $178(80.5 \%)$ & .68 \\
\hline Diabetes & $13(13.0 \%)$ & $21(27.3 \%)$ & $5(23.8 \%)$ & $2(15.4 \%)$ & $1(10.0 \%)$ & $42(19.0 \%)$ & .15 \\
\hline Hyperlipidemia & $74(74.0 \%)$ & $57(74.0 \%)$ & $14(66.7 \%)$ & $8(61.5 \%)$ & $7(70.0 \%)$ & $160(72.4 \%)$ & .85 \\
\hline CAD & $21(21.0 \%)$ & $18(23.4 \%)$ & $7(33.3 \%)$ & $3(23.1 \%)$ & $3(30.0 \%)$ & $52(23.5 \%)$ & .79 \\
\hline PVD & $13(13.0 \%)$ & $12(15.6 \%)$ & $0(0 \%)$ & $0(0 \%)$ & $10(100 \%)$ & $26(11.8 \%)$ & .22 \\
\hline COPD & $27(27.0 \%)$ & $21(27.3 \%)$ & $5(23.8 \%)$ & $1(7.7 \%)$ & $3(30.0 \%)$ & $57(25.8 \%)$ & .64 \\
\hline CVA & $5(5.0 \%)$ & $9(11.7 \%)$ & $2(9.5 \%)$ & $2(15.4 \%)$ & $0(0 \%)$ & $18(8.1 \%)$ & .35 \\
\hline CKD & $8(8.0 \%)$ & $14(18.2 \%)$ & $3(14.3 \%)$ & $1(7.7 \%)$ & $1(10.0 \%)$ & $27(12.2 \%)$ & .33 \\
\hline Carotid stenosis & $1(1.0 \%)$ & $2(2.6 \%)$ & $2(9.5 \%)$ & $1(7.7 \%)$ & $1(10.0 \%)$ & $7(3.2 \%)$ & .15 \\
\hline Arrhythmia & $29(29.0 \%)$ & $41(53.2 \%)$ & $11(52.4 \%)$ & $4(30.8 \%)$ & $1(10.0 \%)$ & $86(38.9 \%)$ & .64 \\
\hline MI & $8(8.0 \%)$ & $5(6.5 \%)$ & $0(0 \%)$ & $2(15.4 \%)$ & $0(0 \%)$ & $15(6.8 \%)$ & .40 \\
\hline LVEF (\%) & $60(57-65)$ & $61(55-65)$ & $60(56-64)$ & $63(59-69)$ & $57(51-61)$ & $56(60-65)$ & .53 \\
\hline $\mathrm{CHF}$ & $8(8.0 \%)$ & $7(9.1 \%)$ & $2(9.5 \%)$ & $2(15.4 \%)$ & $0(0 \%)$ & $19(8.6 \%)$ & .77 \\
\hline NYHA class I & $1(1.0 \%)$ & $6(7.8 \%)$ & $1(4.8 \%)$ & $1(7.7 \%)$ & $0(0 \%)$ & $9(4.1 \%)$ & .20 \\
\hline NYHA class II & $4(4.0 \%)$ & $0(0 \%)$ & $1(4.8 \%)$ & $0(0 \%)$ & $0(0 \%)$ & $5(2.3 \%)$ & .37 \\
\hline NYHA class III & $3(3.0 \%)$ & $1(1.3 \%)$ & $0(0 \%)$ & $1(7.7 \%)$ & $0(0 \%)$ & $5(2.3 \%)$ & .55 \\
\hline NYHA class IV & $0(0 \%)$ & $0(0 \%)$ & $0(0 \%)$ & $0(0 \%)$ & $0(0 \%)$ & $0(0 \%)$ & NA \\
\hline Hemodialysis & $1(1.0 \%)$ & $1(1.3 \%)$ & $0(0 \%)$ & $0(0 \%)$ & $0(0 \%)$ & $2(0.9 \%)$ & .97 \\
\hline Prior PCI & $9(9.0 \%)$ & $4(5.2 \%)$ & $2(9.5 \%)$ & $0(0 \%)$ & $1(10.0 \%)$ & $16(7.2 \%)$ & .69 \\
\hline Prior cardiac surgery & $9(9.0 \%)$ & $9(11.7 \%)$ & $2(9.5 \%)$ & $0(0 \%)$ & $1(10 \%)$ & $21(9.5 \%)$ & .67 \\
\hline
\end{tabular}

$C A D$, Coronary artery disease; $P V D$, peripheral vascular disease; $C O P D$, chronic obstructive pulmonary disease; $C V A$, cerebrovascular accident; $C K D$, chronic kidney disease; $M I$, myocardial infarction; $L V E F$, left ventricular ejection fraction; $C H F$, congestive heart failure; $N Y H A$, New York Heart Association; $N A$, not available; $P C I$, percutaneous coronary intervention.

to $4.9 \mathrm{~cm} \mathrm{(503} \mathrm{[453-569]} \mathrm{vs} 330$ [296-359] $\mathrm{kPa}, P=2.5 \mathrm{e}-$ $14)$, size 5.0 to $5.4 \mathrm{~cm}(549$ [430-569] vs 339 [320-373] $\mathrm{kPa}, P=9.5 \mathrm{e}-7)$, size 5.5 to $5.9 \mathrm{~cm}(540[471-608] \mathrm{vs}$ 318 [293-351] kPa, $P=.0002$ ), and size $6.0 \mathrm{~cm}$ or greater (596 [506-649] vs 373 [363-449] kPa, $P=.002$ ).

Reproducibility of wall stress results was tested with 2 investigators independently reconstructing TAV-aTAA models and performing FE simulations for a $11 \%$ subset of patients $(n=24)$ of the current study. Circumferential TAV-aTAA peak wall stresses were 486 (449-524) $\mathrm{kPa}$ versus 477 (427-521) $\mathrm{kPa}$ for investigators 1 and 2, respectively $(P=.09)$. Longitudinal peak wall stresses were 329 (298-341) $\mathrm{kPa}$ versus 306 (262-345) $\mathrm{kPa}$ for investigator 1 and 2 , respectively $(P=.24)$.

\section{Impact of Blood Pressure Reduction on Tricuspid Aortic Valve Ascending Thoracic Aortic Aneurysm Wall Stresses}

For a subset of TAV-aTAAs $(\mathrm{n}=96)$ with a median diameter $4.4(4.1-4.8) \mathrm{cm}$, overall peak circumferential

TABLE 2. Circumferential tricuspid aortic valve ascending thoracic aortic aneurysm peak wall stress

\begin{tabular}{lcccccc}
\hline & $\mathbf{4 . 0 - 4 . 4} \mathbf{~ c m}(\mathbf{n}=\mathbf{1 0 0})$ & $\mathbf{4 . 5 - 4 . 9} \mathbf{~ c m}(\mathbf{n}=\mathbf{7 7})$ & $\mathbf{5 . 0 - 5 . 4} \mathbf{~ c m}(\mathbf{n}=\mathbf{2 1})$ & $\mathbf{5 . 5 - 5 . 9} \mathbf{~ c m}(\mathbf{n}=\mathbf{1 3})$ & $\geq \mathbf{6 . 0} \mathbf{~ c m}(\mathbf{n}=\mathbf{1 0})$ & $\boldsymbol{P}$ value \\
\hline Whole aTAA $(\mathrm{kPa})$ & $460(421-543)$ & $503(453-569)$ & $549(430-588)$ & $540(471-608)$ & $596(506-649)$ & .0008 \\
SOV $(\mathrm{kPa})$ & $377(334-432)$ & $429(385-488)$ & $424(371-453)$ & $454(415-499)$ & $552(516-627)$ & $1.6 \mathrm{e}-8$ \\
STJ $(\mathrm{kPa})$ & $449(388-501)$ & $512(464-589)$ & $534(439-554)$ & $626(541-754)$ & $573(503-622)$ & $1.3 \mathrm{e}-8$ \\
AscAo $(\mathrm{kPa})$ & $316(290-340)$ & $347(322-365)$ & $358(342-415)$ & $424(400-462)$ & $413(389-466)$ & $2.6 \mathrm{e}-15$ \\
\hline
\end{tabular}

aTAA, Ascending thoracic aortic aneurysm; SOV, sinuses of Valsalva; STJ, sinotubular junction; AscAo, ascending aorta. 

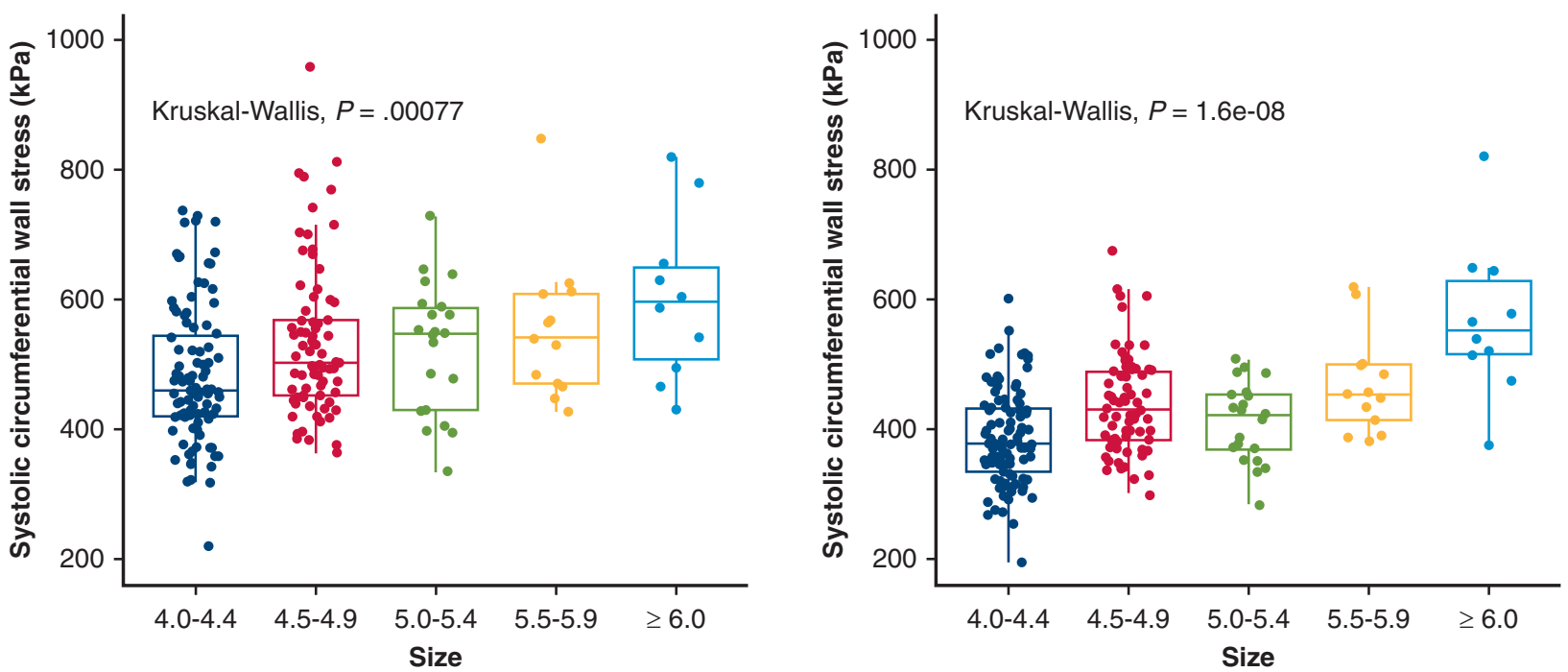

A

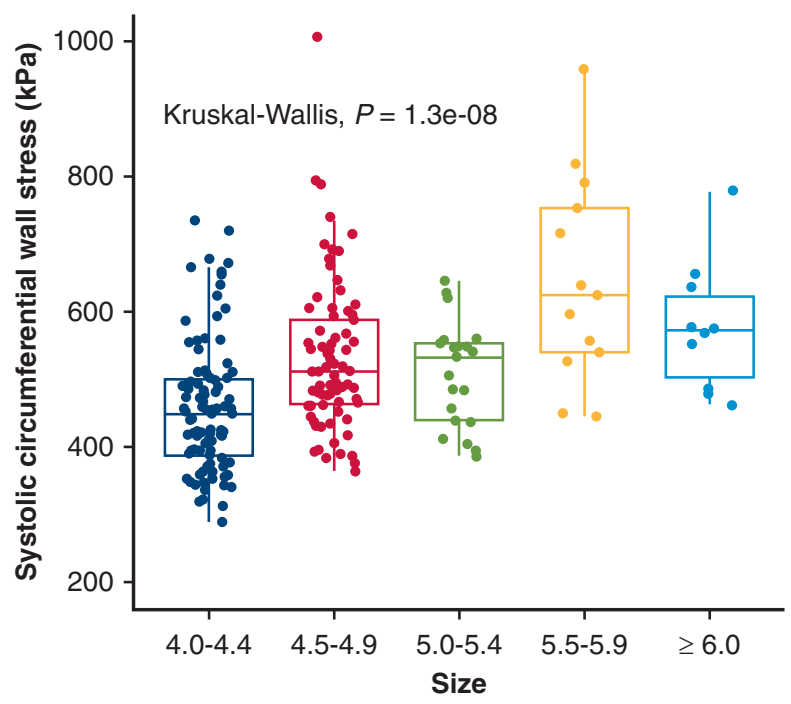

B

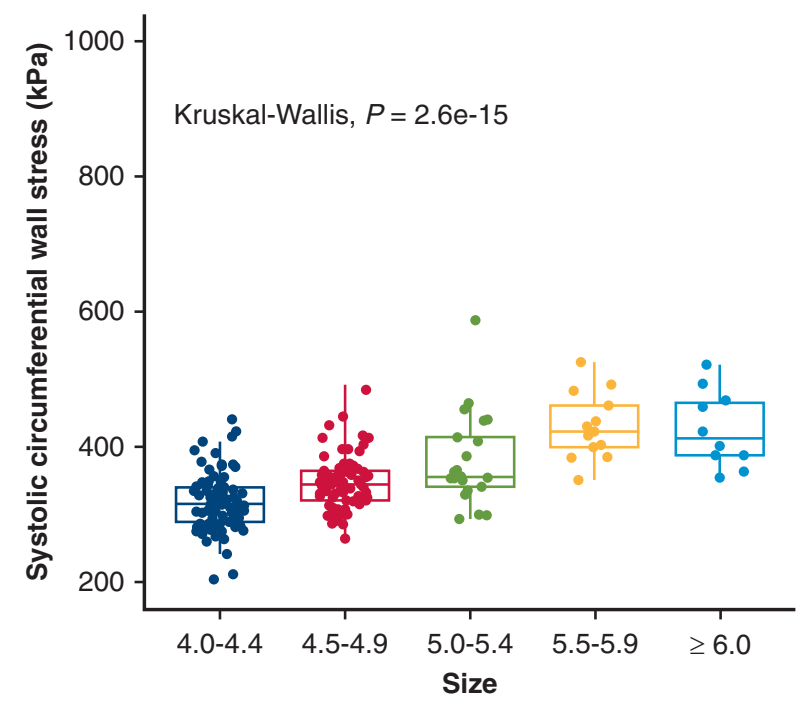

D

FIGURE 2. Relationship between peak circumferential wall stress and diameter group for whole aneurysm (A), SOV (B), STJ (C), and AscAo (D) in TAVaTAA. Median (horizontal line), 25th and 75th percentiles (box), range (whiskers), and observations (dots).

wall stresses were greater at a blood pressure of $120 \mathrm{~mm}$ $\mathrm{Hg}$ than $95 \mathrm{~mm} \mathrm{Hg}$ (444 [385-511] vs 358 [316-412] $\mathrm{kPa}, P<2.2 \mathrm{e}-16)$. Peak longitudinal wall stresses were also greater at a blood pressure of $120 \mathrm{~mm} \mathrm{Hg}$ than $95 \mathrm{~mm} \mathrm{Hg}(283$ [257-312] vs 238 [213-260] kPa, $P<2.2 \mathrm{e}-16)$.

\section{DISCUSSION}

In this study, we found that the magnitudes of TAV-aTAA peak wall stress increase with diameter in both circumferential and longitudinal directions. However, aTAA diameter groups had a wide distribution of stresses resulting in a large overlap between different groups. The same findings

TABLE 3. Longitudinal tricuspid aortic valve ascending thoracic aortic aneurysm peak wall stress

\begin{tabular}{lcccccc}
\hline & $\mathbf{4 . 0 - 4 . 4} \mathbf{~ c m ~}(\mathbf{n}=\mathbf{1 0 0})$ & $\mathbf{4 . 5 - 4 . 9} \mathbf{~ c m}(\mathbf{n}=\mathbf{7 7})$ & $\mathbf{5 . 0 - 5 . 4} \mathbf{~ c m}(\mathbf{n}=\mathbf{2 1})$ & $\mathbf{5 . 5 - 5 . 9} \mathbf{~ c m}(\mathbf{n}=\mathbf{1 3})$ & $\geq \mathbf{6 . 0} \mathbf{~ c m}(\mathbf{n}=\mathbf{1 0})$ & $\boldsymbol{P}$ value \\
\hline Whole aTAA $(\mathrm{kPa})$ & $290(265-323)$ & $330(296-359)$ & $339(320-373)$ & $318(293-351)$ & $373(363-449)$ & $8.7 \mathrm{e}-8$ \\
SOV $(\mathrm{kPa})$ & $295(263-333)$ & $337(307-366)$ & $339(317-380)$ & $359(320-437)$ & $435(421-463)$ & $6.6 \mathrm{e}-10$ \\
STJ $(\mathrm{kPa})$ & $206(184-251)$ & $255(223-286)$ & $284(229-299)$ & $318(277-349)$ & $330(299-413)$ & $2.1 \mathrm{e}-11$ \\
AscAo $(\mathrm{kPa})$ & $176(157-193)$ & $194(182-213)$ & $225(203-245)$ & $227(218-251)$ & $248(223-256)$ & $8.5 \mathrm{e}-13$ \\
\hline
\end{tabular}

aTAA, Ascending thoracic aortic aneurysm; SOV, sinuses of Valsalva; STJ, sinotubular junction; AscAo, ascending aorta. 

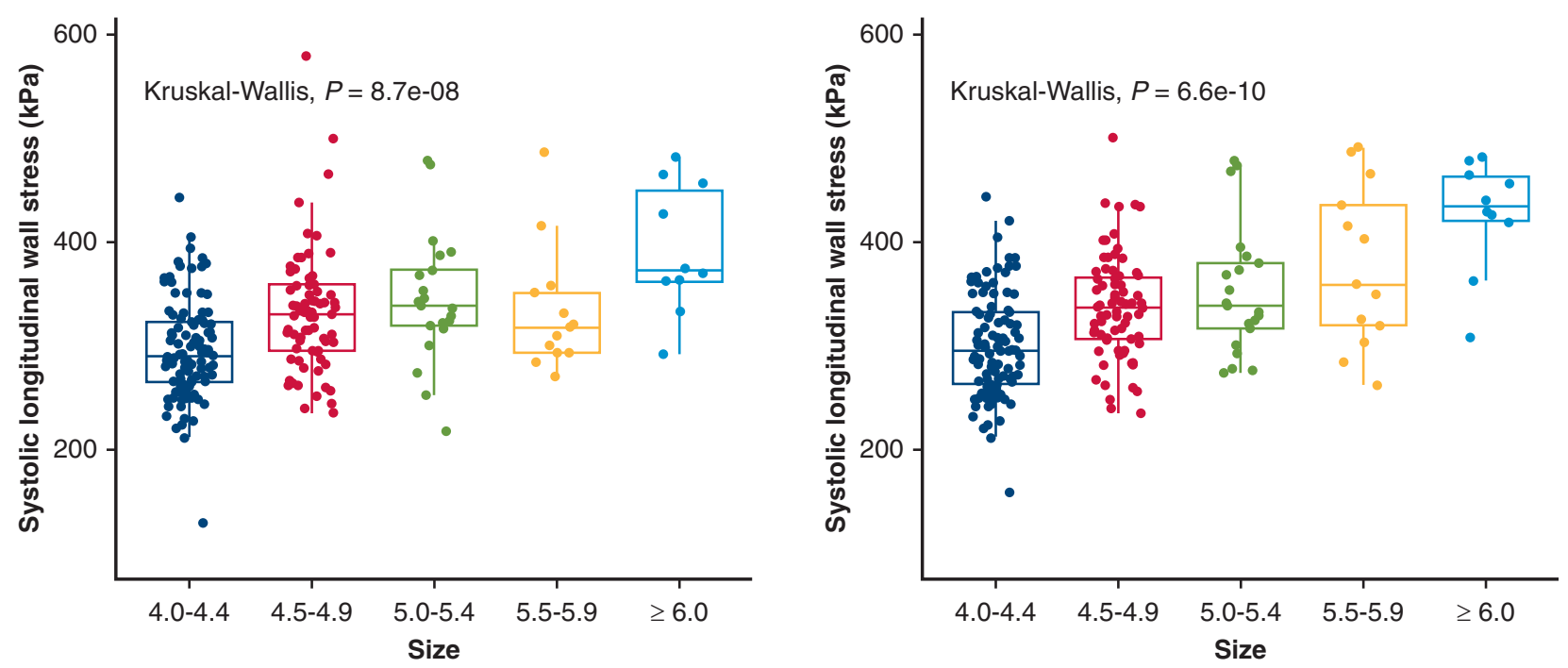

A

B
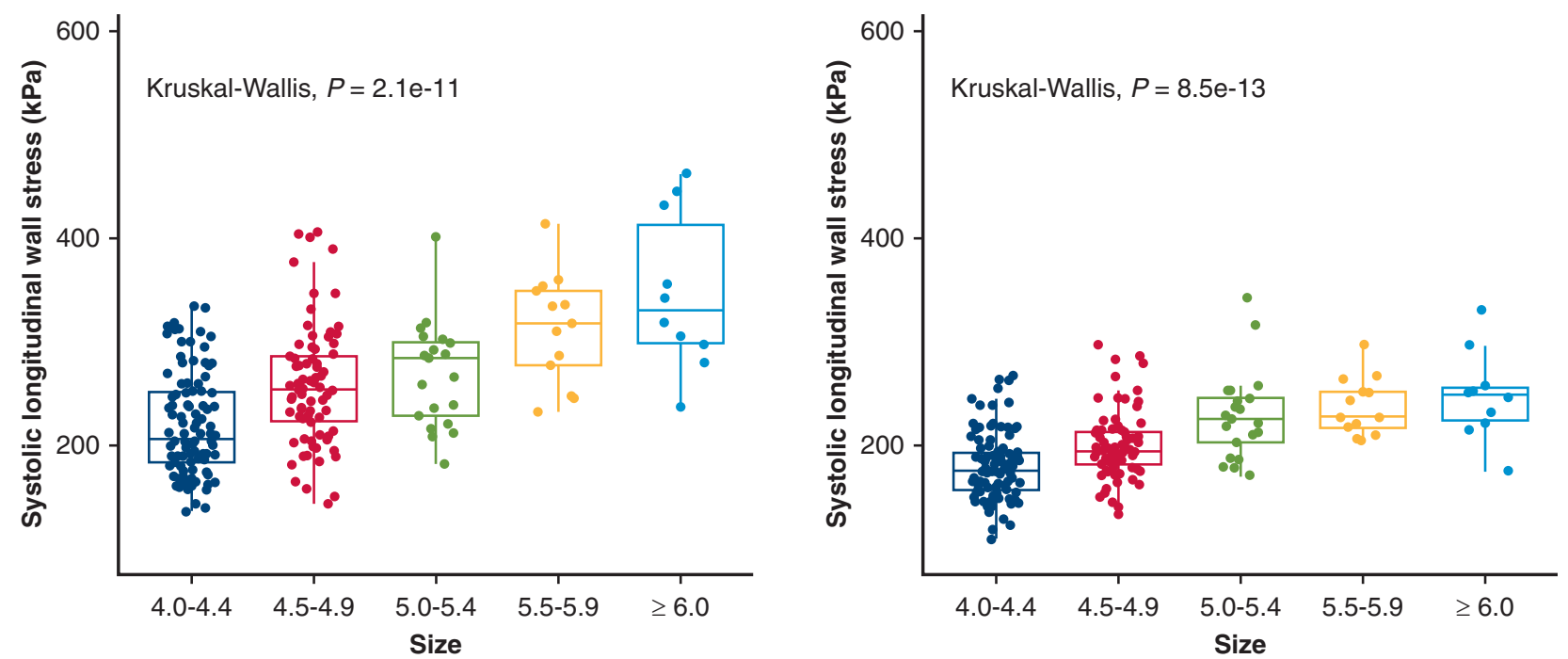

C

\section{D}

FIGURE 3. Relationship between peak longitudinal wall stress and diameter group for whole aneurysm (A), SOV (B), STJ (C), and AscAo (D) in TAVaTAA. Median (horizontal line), 25th and 75th percentiles (box), range (whiskers), and observations (dots).

remained true when considering stresses limited to the SOV, STJ, or AscAo. These findings are consistent with the concept that dissection risk increases with diameter in a population level. However, we found that even small aneurysms can experience wall stresses as large as aneurysms that met surgical size criteria for operation, and some small aneurysms may have large enough wall stress magnitudes to predispose to acute aortic events. These aTAA cases would otherwise have been deemed to be at low risk of dissection or rupture based on diameter alone. Conversely, some patients who would be offered surgical repair based on diameter-based surgical guidelines had wall stresses that were so low and similar to small size aneurysms that they may not be predisposed to dissection.

\section{Finite Element Analyses Studies in Literature}

Our findings are consistent with other smaller finite element studies in the literature. Trabelsi and colleagues ${ }^{17}$ reported that peak wall stress was only loosely correlated with diameter $(\rho=0.53)$ in a cohort of 5 patients with patient-specific material properties. ${ }^{17}$ Of interest, the rupture risk index, defined as the ratio of peak to failure stress, was not positively correlated with diameter $(\rho=-0.29)$, suggesting diameter is inadequate to predict 


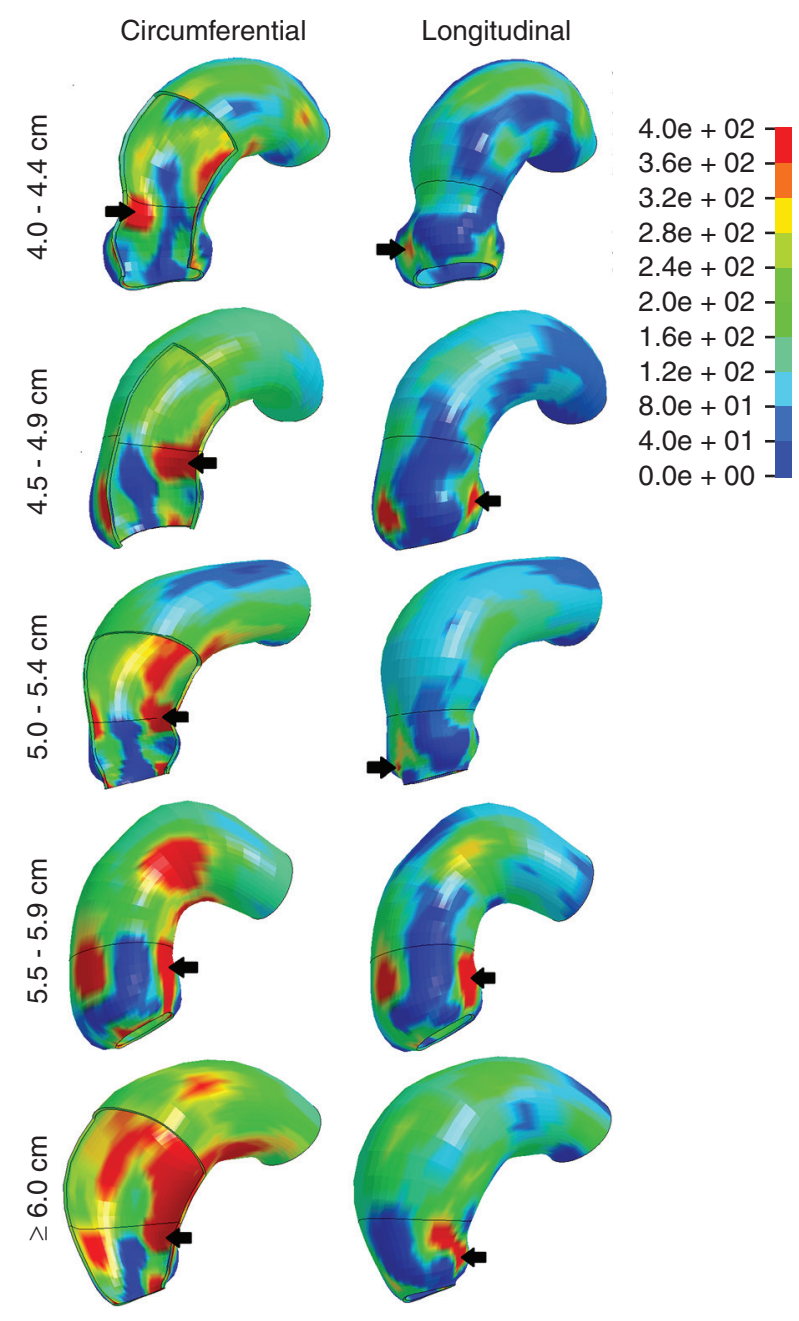

FIGURE 4. Characteristic TAV-aTAA wall stress profiles by diameter group in both circumferential and longitudinal directions. Color bar displays the relation of figure colors to wall stress magnitude in $\mathrm{kPa}$. Location of highest wall stress are indicated by a black arrow.

dissection. Martin and colleagues ${ }^{18}$ found that peak systolic wall stress was unrelated to diameter $(P=.08)$ in a group of 27 aneurysms. Notably, they found the diameter risk ratio (defined as aTAA diameter at systole over diameter preceding failure), a metric to capture dissection/rupture risk, missed any observable relation with maximum diameter, but correlated instead with peak wall stress. These findings support wall stress as a superior predictor of dissection or aortic events. Nonetheless, both of these studies were limited by a small sample size, which increases the risk of type II error. Both studies also prevented secondary analysis of TAV-aTAAs exclusively. Similar findings have been described by our group. In an early study, we observed that in TAV-aTAAs, both circumferential and longitudinal systolic wall stresses had moderate correlations with diameter $(\mathrm{r}=0.71$ and 0.68 , respectively). These correlations were stronger than their bicuspid counterparts. ${ }^{9}$ This study was also limited by a small sample size $(\mathrm{n}=18)$ and aneurysm geometries that excluded SOV and LVOT potentially influencing wall stress values based upon boundary conditions. Our subsequent study with approximately 100 aTAAs addressed these limitations and found a correlation of wall stress with diameter that was improved for large aneurysms size $5 \mathrm{~cm}$ or greater than those less than $5 \mathrm{~cm} .{ }^{12}$ However, the correlation was poor to moderate in either group. This observation remained true when studying TAV-aTAAs alone. This study corroborates our findings with a larger study focused on TAV-aTAAs. Overall peak aTAA wall stresses correlated poorly with diameter with $\mathrm{r}$ correlation coefficients of 0.26 and 0.38 for circumferential and longitudinal directions, respectively. Similar to our new findings, our previous study demonstrated a large overlap of stresses between less than $5 \mathrm{~cm}$ and $5 \mathrm{~cm}$ or greater aTAAs. Our current FE study adds to our prior work by examining a larger cohort of aTAAs, double the prior study, and specifically focused on TAV-associated aTAAs $(n=221)$ to examine diameters incrementally by every $0.5 \mathrm{~cm}$ and understand the impact of the $5.5 \mathrm{~cm}$ diameter cutoff for aneurysm repair on wall stresses. Of interest, studies have previously proposed and evaluated index diameter metrics including diameter/height and diameter/body surface area as patient-specific predictors of aneurysm dissection and rupture. ${ }^{3}$ On the basis of these updated guidelines, future studies could evaluate if index diameter metrics provide a better correlation with wall stress.

\section{Biomechanics of Acute Type A Aortic Dissection}

In theory from a biomechanical standpoint, aortic dissection may occur from failure of the aortic intimal layer due to high wall stresses versus wall strength. ${ }^{7}$ Wall strength cannot be determined in vivo as it requires destructive tissue testing from human aTAA surgical specimens. An alternative would be to assess the relationship between in vivo distensibility (aortic stiffness) and ex vivo failure mechanics. Pichamuthu and colleagues ${ }^{19}$ studied the failure mechanics of TAV versus BAV-ATAAs with uniaxial tensile testing. BAVaTAA wall strength was found to be greater than that of TAV-aTAA in both circumferential and longitudinal directions. Others found that BAV-aTAA strength was equal to stronger than TAV-aTAAs. ${ }^{20-22}$ In both BAV and TAV aneurysms, circumferential tensile strength was approximately twice the strength in the longitudinal direction. Although circumferential stresses are significantly greater than longitudinal stresses, the lower longitudinal strength in relationship to circumferential strength explains the reason why dissection entry tears begin transversely. ${ }^{23}$ Gleason's group ${ }^{19}$ reported a longitudinal tensile strength of $54 \mathrm{~N} / \mathrm{cm}^{2}(540 \mathrm{kPa})$. Tensile strength in relation to wall stress could estimate dissection or rupture risk. One of our small aTAAs with size $4.5 \mathrm{~cm}$ already 
exceeded this strength value. Notably, our results were calculated assuming normal blood pressure $(120 / 80 \mathrm{~mm} \mathrm{Hg})$. However, hypertension of varying severity was prevalent $(80 \%)$ in our cohort. Using a quick nonpatient-specific calculation based on Laplace's law, patients with aTAAs with peak longitudinal wall stress greater than $462 \mathrm{kPa}$ could reach a stress of $540 \mathrm{kPa}$ when experiencing a systolic pressure of $140 \mathrm{~mm} \mathrm{Hg}$. Applied to our cohort, this would include $5 \%$ of aTAAs size 4.5 to $5.4 \mathrm{~cm}$ and $18 \%$ of size $5.5 \mathrm{~cm}$ or greater. Close follow-up and prospective investigation of patients with approximately $462 \mathrm{kPa}$ longitudinal stress might be considered to assess a potential surgical wall stress threshold. In this study, we also showed for a group of small aneurysms that blood pressure reduction from 120 to $95 \mathrm{~mm} \mathrm{Hg}$ resulted in a $20 \%$ reduction in longitudinal wall stresses. A $20 \%$ wall stress reduction decreased peak longitudinal wall stresses to less than $462 \mathrm{kPa}$ in all but one of our aTAAs less than $5.5 \mathrm{~cm}$. These results emphasize the benefit of blood pressure reduction as a means to reduce aneurysm wall stress and risk of dissection. Although the complexity of dissection may involve the patterns of blood pressure and tissue remodeling over time, the beauty of patient-specific aTAA biomechanics is that as long as the parameters are known, such as changes in blood pressure, aTAA geometry, and in vivo distensibility (as a surrogate for wall strength), then patient-specific simulations over time could provide understanding of when an individual patient transitions from low to higher risk of dissection.

We could also apply epidemiological rates of dissection to our cohort as another means of estimating a surgical stress threshold. Based on a cohort of approximately 800 aTAAs, Zafar and colleagues ${ }^{3}$ reported annual type A dissection rates for the same size groups we used in the current study and found dissection rates of $1.2 \%$ for size 4.0 to $4.4 \mathrm{~cm}, 2.0 \%$ for 4.5 to $4.9 \mathrm{~cm}, 1.8 \%$ for 5.0 to $5.4 \mathrm{~cm}$, $3.5 \%$ for 5.5 to $5.9 \mathrm{~cm}$, and $9.6 \%$ for $6.0 \mathrm{~cm}$ or greater. ${ }^{3}$ By matching dissection rates to corresponding percentiles of longitudinal wall stress in the same diameter groups, we hypothesize that TAV-aTAAs with longitudinal peak wall stress of at least $457 \mathrm{kPa}$ would be at high risk of dissection events. The percentage of TAV-aTAAs with peak longitudinal wall stresses above this stress threshold were $0 \%$ for size 4.0 to $4.4 \mathrm{~cm}, 3.9 \%$ for 4.5 to $4.9 \mathrm{~cm}$, $9.5 \%$ for 5.0 to $5.4 \mathrm{~cm}, 7.7 \%$ for 5.5 to $5.9 \mathrm{~cm}$, and $30 \%$ for $6.0 \mathrm{~cm}$ or greater in our cohort. A surgical wall stress threshold of approximately $450 \mathrm{kPa}$ would be congruent with both the epidemiologic dissection data and failure data available at present. ${ }^{24-26}$ To better define a surgical threshold, a comprehensive biomechanical study of wall failure properties in relation to the wall stresses specific to aTAA considering all aortic subregions would be beneficial.

Of interest, a recent study found that aTAAs that dissected and had predissection imaging $(n=7)$, experienced higher longitudinal wall stresses than age and size-matched controls with the location of the entry tear matching regions of increased stress. ${ }^{27}$ In our study, we found that a group of TAV-aTAAs less than $5.5 \mathrm{~cm}$ experience longitudinal wall stresses similar in magnitude to aneurysms $5.5 \mathrm{~cm}$ or greater. These findings remained true when studying aneurysms as a whole or by specific aortic regions (ie, STJ, SOV, AscAo). Celi and Berti ${ }^{28}$ noted that small aneurysms can have large stress values when aneurysm eccentricity is present. Taken together, these observations have the following clinical implications. ATAA wall stresses are dependent on the whole 3D geometry rather than a single maximal diameter value. Small aneurysms that experience large longitudinal stresses could be at high risk of dissection explaining the acute events that occur in aneurysms not meeting surgical repair guidelines. ${ }^{5,6,29}$ Determining patient-specific TAV-aTAA biomechanics through computational models may further help determine dissection risk and need for elective aTAA surgery despite less than $5.5 \mathrm{~cm}$ size.

The use of FEA and wall stresses as tools to assist surgical decision making will require prospective validation in clinical studies. In the present study, we have shown that diameter is poorly correlated to wall stress and that the law of LaPlace is inadequate in these complex geometries. The underlying natural history studies from which the guidelines were based used diameter as a surrogate for wall stress and developed the size thresholds using patients who had already dissected. The limited studies that have captured predissection diameters in patients whose imaging was performed within 2 years of dissection have shown diameters in the 4.3 - to $4.5-\mathrm{cm}$ range. ${ }^{27,29}$ As such, a prospective study of patients with nonsurgical sized aneurysms followed regularly for clinical events and correlated with not only wall stresses but also diameter and other parameters is needed to fundamentally improve on existing guidelines. Although we did not show a correlation of wall stresses to clinical events in this study, Emerel and colleagues $^{27}$ demonstrated that increased longitudinal wall stresses correlated with intimal tear location. Thus, our study requires future patient follow-up for clinical events.

\section{Study Limitations}

Our cohort is heavily weighted to the male population reflective of the veteran population. Ninety-eight percent of our subjects were men with a long history of smoking and high rates of hypertension, hyperlipidemia, and other cardiovascular risk factors. These results may not be reflective of women or civilian populations with different risk profiles. Although our limitation is that the relation of wall stress and dissection risk is not directly established, others have shown a direct correlation of increased longitudinal wall stresses with intimal tear in dissection. ${ }^{27}$ Our study requires continued patient follow-up for clinical events. In addition, our model has multiple assumptions. 
TAV-aTAA was assumed to be homogenous, while regional variations in thickness and mechanical properties do exist. Current CT modalities are insufficient to accurately resolve aTAA wall thickness but are the most commonly used imaging techniques. Even cine magnetic resonance imaging, which we have used in a few patients to resolve wall thickness only for research purposes, measures thickness at 1 plane rather than through the entire aTAA region. Thickness used here was based on average ex vivo aTAA tissue thickness, which upon FEA simulation to systemic pressure provided in vivo aTAA wall thickness. Average TAV-ATAA material properties were used, which provide reasonable estimation of patient-specific wall stresses since we demonstrated previously that there were no significant differences in FEA results among simulations using patient-specific, group-averaged, and population-based literature material properties. ${ }^{30}$ Whole wall properties were used, and the impact of incorporating differences in wall layers material properties $^{27}$ can be explored in the future. Longitudinal motion was fixed at the LVOT and mid-thoracic descending aorta while cardiac motion during the cardiac cycle does occur. These assumptions can influence simulation results. The present study did not consider fluid-structure interactions or blood flow patterns such as higher velocity and skewed retrograde and helical flow of aneurysmal aorta, which was beyond the scope of this project. ${ }^{31,32}$ Wall shear stresses were not considered because they are 5 orders of magnitude lower than wall stress due to blood pressure with respect to dissection or rupture risk. ${ }^{33}$ Wall shear stresses and helical flows are most commonly perturbed in BAV-aTAA, and that population was excluded in this study.

\section{CONCLUSIONS}

We described patient-specific TAV-aTAA wall stresses by size and found that circumferential and longitudinal stresses increase with diameter with a large overlap of ranges between small and large aneurysms. Some of our patients with small aTAA are potentially at higher dissection risk, consistent with studies reporting large proportion of dissection events occurring in patients with aneurysms less than $5.5 \mathrm{~cm}$. Wall stresses were effectively reduced with blood pressure reduction, emphasizing the importance of medical therapy to reduce risk. This study highlights the potential utility of patient-specific aTAA wall stresses in relation to wall strength to understand dissection risk and assist decision-making of elective surgical aTAA repair.

\section{Conflict of Interest Statement}

The authors reported no conflicts of interest.

The Journal policy requires editors and reviewers to disclose conflicts of interest and to decline handling or reviewing manuscripts for which they may have a conflict of interest. The editors and reviewers of this article have no conflicts of interest.

\section{References}

1. Guo MH, Appoo JJ, Saczkowski R, Smith HN, Ouzounian M, Gregory AJ, et al Association of mortality and acute aortic events with ascending aortic aneurysm: a systematic review and meta-analysis. JAMA Netw Open. 2018;1:e181281.

2. Chiappini B, Schepens M, Tan E, Dell' Amore A, Morshuis W, Dossche K, et al. Early and late outcomes of acute type A aortic dissection: analysis of risk factors in 487 consecutive patients. Eur Heart J. 2005;26:180-6.

3. Zafar MA, Li Y, Rizzo JA, Charilaou P, Saeyeldin A, Velasquez CA, et al. Height alone, rather than body surface area, suffices for risk estimation in ascending aortic aneurysm. J Thorac Cardiovasc Surg. 2018;155:1938-50.

4. Hiratzka LF, Bakris GL, Beckman JA, Bersin RM, Carr VF, Casey DE, et al. 2010 ACCF/AHA/AATS/ACR/ASA/SCA/SCAI/SIR/STS/SVM guidelines for the diagnosis and management of patients with thoracic aortic disease: a report of the American College of Cardiology Foundation/American Heart Association task force on practice guidelines, American Association for Thoracic Surgery, American College of Radiology, American Stroke Association, Society of Cardiovascular Anesthesiologists, Society for Cardiovascular Angiography and Interventions, Society of Interventional Radiology, Society of Thoracic Surgeons, and Society for Vascular Medicine. Circulation. 2010;121: e266-369.

5. Pape LA, Tsai TT, Isselbacher EM, Oh JK, O'gara PT, Evangelista A, et al. Aortic diameter $>$ or $=5.5 \mathrm{~cm}$ is not a good predictor of type A aortic dissection: observations from the International Registry of Acute Aortic Dissection (IRAD). Circulation. 2007;116:1120-7.

6. Jaussaud N, Chitsaz S, Meadows A, Wintermark M, Cambronero N, Azadani AN, et al. Acute type A aortic dissection intimal tears by 64-slice computed tomography: a role for endovascular stent-grafting? J Cardiovasc Surg (Torino). 2013;54:373-81

7. Vorp DA, Schiro BJ, Ehrlich MP, Juvonen TS, Ergin MA, Griffith BP. Effect of aneurysm on the tensile strength and biomechanical behavior of the ascending thoracic aorta. Ann Thorac Surg. 2003;75:1210-4.

8. Nathan DP, Xu C, Gorman JH, Fairman RM, Bavaria JE, Gorman RC, et al. Pathogenesis of acute aortic dissection: a finite element stress analysis. Ann Thorac Surg. 2011;91:458-63.

9. Xuan Y, Wang Z, Liu R, Haraldsson H, Hope MD, Saloner DA, et al. Wall stress on ascending thoracic aortic aneurysms with bicuspid compared with tricuspid aortic valve. J Thorac Cardiovasc Surg. 2018;156:492-500.

10. Plonek T, Zak M, Rylski B, Berezowski M, Czerny M, Beyersdorf F, et al. Wall stress correlates with intimal entry tear localization in Type A aortic dissection. Interact Cardiovasc Thorac Surg. 2018;27:797-801.

11. Gomez A, Wang Z, Xuan Y, Wisneski AD, Hope MD, Saloner DA, et al. Wall stress distribution in bicuspid aortic valve-associated ascending thoracic aortic aneurysms. Ann Thorac Surg. 2020;110:807-14.

12. Wang Z, Flores N, Lum M, Wisneski AD, Xuan Y, Inman J, et al. Wall stress analyses in patients with $\geq 5 \mathrm{~cm}$ versus $<5 \mathrm{~cm}$ ascending thoracic aortic aneurysm. $J$ Thorac Cardiovasc Surg. February 19, 2020 [Epub ahead of print].

13. Gee MW, Förster C, Wall WA. A computational strategy for prestressing patientspecific biomechanical problems under finite deformation. Int J Numer Method Biomed Eng. 2010;26:52-72.

14. Krishnan K, Ge L, Haraldsson H, Hope MD, Saloner DA, Guccione JM, et al. Ascending thoracic aortic aneurysm wall stress analysis using patient-specific finite element modeling of in vivo magnetic resonance imaging. Interact Cardiovasc Thorac Surg. 2015;21:471-80.

15. Azadani AN, Chitsaz S, Mannion A, Mookhoek A, Wisneski A, Guccione JM, et al. Biomechanical properties of human ascending thoracic aortic aneurysms. Ann Thorac Surg. 2013;96:50-8.

16. Speelman L, Bosboom EM, Schurink GW, Hellenthal FA, Buth J, Breeuwer M, et al. Patient-specific AAA wall stress analysis: 99-percentile versus peak stress. Eur J Vasc Endovasc Surg. 2008;36:668-76.

17. Trabelsi O, Davis FM, Rodriguez-Matas JF, Duprey A, Avril S. Patient specific stress and rupture analysis of ascending thoracic aneurysms. J Biomech. 2015; 48:1836-43.

18. Martin C, Sun W, Elefteriades J. Patient-specific finite element analysis of ascending aorta aneurysms. Am J Physiol Heart Circ Physiol. 2015;308: H1306-16.

19. Pichamuthu JE, Phillippi JA, Cleary DA, Chew DW, Hempel J, Vorp DA, et al. Differential tensile strength and collagen composition in ascending aortic aneurysms by aortic valve phenotype. Ann Thorac Surg. 2013;96:2147-54. 
20. Deveja RP, Iliopoulos DC, Kritharis EP, Angouras DC, Sfyris D, Papadodima SA, et al. Effect of aneurysm and bicuspid aortic valve on layer-specific ascending aorta mechanics. Ann Thorac Surg. 2018;106:1692-701.

21. Pham T, Martin C, Elefteriades J, Sun W. Biomechanical characterization of ascending aortic aneurysm with concomitant bicuspid aortic valve and bovine aortic arch. Acta Biomater. 2013:9:7927-36.

22. Duprey A, Trabelsi O, Vola M, Favre JP, Avril S. Biaxial rupture properties of ascending thoracic aortic aneurysms. Acta Biomater. 2016;42:273-85.

23. Ge L, Tseng EE. Invited Commentary. Ann Thorac Surg. 2013;96:2154.

24. Iliopoulos DC, Deveja RP, Kritharis EP, Perrea D, Sionis GD, Toutouzas K, et al. Regional and directional variations in the mechanical properties of ascending thoracic aortic aneurysms. Med Eng Phys. 2009;31:1-9.

25. Azadani AN, Chitsaz S, Matthews PB, Jaussaud N, Leung J, Tsinman T, et al. Comparison of mechanical properties of human ascending aorta and aortic sinuses. Ann Thorac Surg. 2012;93:87-94.

26. Sokolis DP, Kritharis EP, Iliopoulos DC. Effect of layer heterogeneity on the biomechanical properties of ascending thoracic aortic aneurysms. Med Biol Eng Comput. 2012;50:1227-37.

27. Emerel L, Thunes J, Kickliter T, Billaud M, Phillippi JA, Vorp DA, et al. Predissection-derived geometric and distensibility indices reveal increased peak longitudinal stress and stiffness in patients sustaining acute type A aortic dissection: Implications for predicting dissection. J Thorac Cardiovasc Surg. 2019;158:355-63.
28. Celi S, Berti S. Three-dimensional sensitivity assessment of thoracic aortic aneurysm wall stress: a probabilistic finite-element study. Eur J Cardiothorac Surg. 2014;45:467-75.

29. Parish LM, Gorman JH, Kahn S, Plappert T, St John-Sutton MG, Bavaria JE, et al. Aortic size in acute type A dissection: implications for preventive ascending aortic replacement. Eur J Cardiothorac Surg. 2009;35:941-6.

30. Wang Z, Yuan X, Guccione JM, Tseng EE, Ge L. Impact of patient-specific material properties on aneurysm wall stress: finite element study. J Heart Valve Dis 2018-2019;27:275-84.

31. Gülan U, Calen C, Duru F, Holzner M. Blood flow patterns and pressure loss in the ascending aorta: a comparative study on physiological and aneurysmal conditions. J Biomech. 2018;76:152-9.

32. Weigang E, Kari FA, Beyersdorf F, Luehr M, Etz CD, Frydrychowicz A, et al. Flow-sensitive four-dimensional magnetic resonance imaging: flow patterns in ascending aortic aneurysms. Eur J Cardiothorac Surg. 2008;34:11-6.

33. Pasta S, Rinaudo A, Luca A, Pilato M, Scardulla C, Gleason TG, et al. Difference in hemodynamic and wall stress of ascending thoracic aortic aneurysms with bicuspid and tricuspid aortic valve. J Biomech. 2013;46:1729-38.

Key Words: ascending thoracic aortic aneurysms, finite element analysis, tricuspid aortic valve 